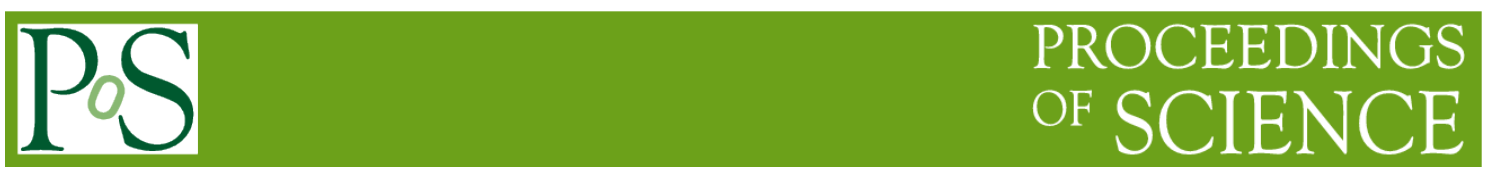

\title{
Mobile Crowd Sensing Networks Technology
}

\author{
Shidong $\mathbf{Y u}^{1}$ \\ College of Software, Shenyang Normal University, Shenyang,110034, China \\ E-mail: ysd0510@sina.com
}

\section{Peng $\mathbf{W u}^{2}$}

College of Software, Shenyang Normal University, Shenyang,110034, China

E-mail: wu.peng@163.com

\section{Hang Li}

College of Software, Shenyang Normal University, Shenyang,110034, China

E-mail: Iihangnet@gmail.com

With the explosive popularization of the wireless mobile terminal devices, the combination of mobile sensing and crowdsourcing brings about a novel sensing paradigm of Internet of Things (IoT). By means of analyzing the data collected from mobile terminal, the crowd sensing can sense user's situation intelligently and provide personalized service. It summarizes the latest researches both at home and aboard, introduces the state of art in mobile crowd sensing from four aspects, namely, the data processing, the motivation mechanism, the crowd sensing applications and the platforms, and also puts forward our opinions in respect of the abovementioned research fields.

CENet2015

12-13 September 2015

Shanghai, China

${ }^{1}$ Speaker

Corresponding Author

${ }^{2}$ In this paper, the research was sponsored by Science Research Project of Education Department of Liaoning, China (Grant No.: L2014440) 


\section{Introduction}

The Internet of things has entered the depth development stage and more intense demand of physical environment with thorough perception. With the development of wireless communication and sensor technology, the market for smart mobile phone, tablet computer, portable device, vehicle sensing device and mobile terminal integrating more and more sensors, computing, sensing, storage and communication ability has become more and more powerful. With the explosion of the wireless mobile terminal devices developed for more than ten years, how to use specific deployment of sensors to provide service, Internet of things by taking advantage of these ubiquitous mobile devices to provide more large-scale, more complex, thorough and comprehensive service has accessed a new development era.

As to the crowd sensing, as a hot area of research, many scholars in data collection, data processing, data transmission, incentive mechanism and application areas have done a lot of research. Based on the development of the crowd sensing network, this paper introduces its concept, basic features and latest researches while putting forward our opinions in respect of the abovementioned research fields.

\section{Basic Concept and Features of the Crowd Sensing Networks}

\subsection{Basic Concept of Crowd Sensing Networks}

The new mode of perception by using ubiquitous mobile devices to provide awareness services in academics generally is called "human-oriented perception". According to the type and scale of perceived objects, this mode of perception can be divided into two categories: personal sensing and community sensing. The Typical applications of personal sensing include individual movement patterns (such as standing, walking, jogging and running etc.) were monitored to promote health while the daily traffic patterns on the individual (such as bicycles, cars, buses and trains) were monitored to record personal carbon footprint and more. By contrast, the community sensing can accomplish large-scale and complex social perception tasks depending on individual that are difficult to achieve, for example, only when a large number of individuals provide the air quality information and speed and pool analysis of this information, the monitoring of air quality and traffic jams in the city application can understand the urban air quality and traffic conditions.

The community sensing is also known as "crowd-sensing"[1-2], which mainly comes from the idea of crowdsourcing; thus it is also called the "crowd sourced sensing", a term invented by Wired magazine in 2006 and used to describe a new distributed problem solving and work mode, in which the companies use the Internet for assignments to find ideas or solve technical problems. In recent years, people have combined the crowdsourcing with the mobile awareness. Ordinary user's mobile devices are used as the basic sensing unit to collaborate consciously or unconsciously by means of the mobile internet, form an intelligence crowd sensing networks and achieve the distributed sensing tasks and data collection to complete large-scale, complex social sensing tasks.

\subsection{Basic Features of Crowd Sensing Networks}

In the traditional wireless sensor networks, people are just "consumers" of the ultimate sensory data. In contrast, the crowd sensing networks highlight one of the most important features that people will be involved in the data awareness, transmission, analysis, application of the whole system of each process, sensory data "consumers" and sensory data "producers" to adopt a popular new word, called the "Prosumer". The basic characteristic of human-oriented has brought unprecedented opportunities for things perception and transmission means, specifically as follows.

1. Network Maintenance Easier

Firstly, the network nodes generally have a better energy supply, more computing, storage and communication capabilities; secondly, these nodes are usually managed and maintained by 
its holder in good working condition. For example, people are always ready to charge their phones and other mobile devices as needed.

\section{Network Deployment Costs Lower}

Firstly, the city has a large number of mobile devices or vehicles without special deployment; secondly, the human mobility can contribute to perceived coverage and data transfer. On the one hand, holders of a mobile device with random access to all parts and the nodes can sense anytime at anywhere; on the other hand, the "store-carry-forward" opportunity transfer mode can be used because of interaction between the mobile nodes to transmit sensor data in intermittent connectivity network environment.

3. System More Scalable

We just need to recruit more users to participate in the expansion of the system to meet the scale of application.

Thanks to these advantages, the crowd sensing networks have become an important Internet-aware tool. The universal mobile sensing devices can be used to complete large-scale social awareness tasks, which rely on individuals are difficult to achieve.

\section{Research on Crowd Sensing Technology}

The crowd sensing model is shown in Fig. 1, including the data collection from many sensor nodes to use these data to the crowd sensing applications by means of data processing technology.

At present, the crowd sensing research field can be simply divided into the following categories: data processing, incentive mechanism, crowd sensing application and crowd sensing platform, etc.

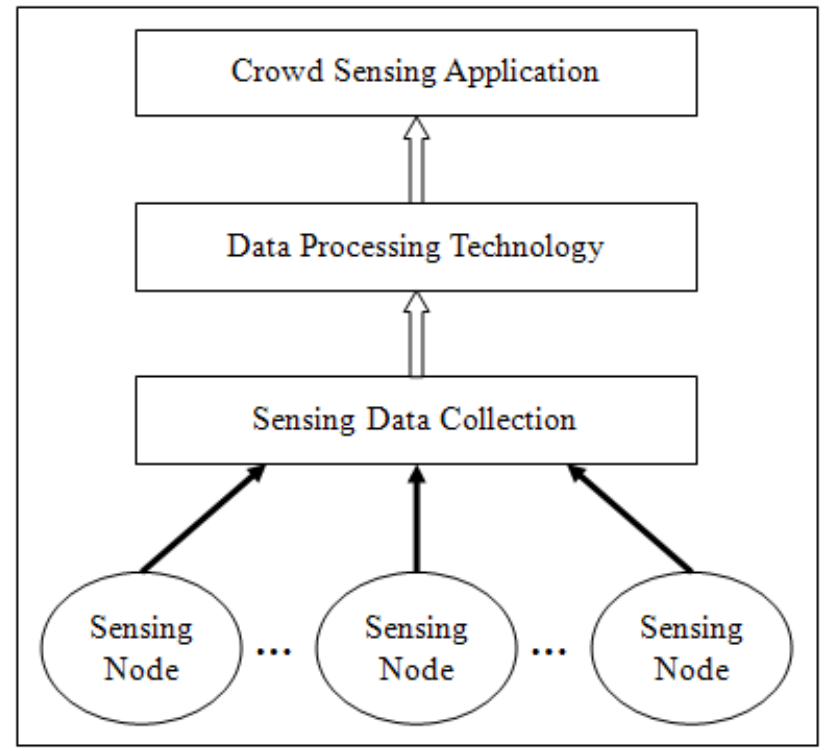

Figure 1 : Crowd Sensing Model

\subsection{Data Processing Technology}

In the crowd sensing, data collection is very important and only the data being utilized is valuable. Data processing, filtering and analysis for large amount of data are crucial for obtaining effective information. Many techniques and tools have provided effective means and ways for the data processing.

Toped ++ is a data conversion interface, similar to the paper filter [3]; besides, there are many interfaces that resemble the entity resolution and pattern matching [4-6]. The data quality and the document structure can refer to Bellman [7]. Potter's wheel ensured the data format and 
singularity point monitoring [8]. The authors introduced the swarm intelligence model on the basis of the model of ant colony optimization algorithm and particle swarm optimization algorithm with detailed as summed up and compared. Currently, the ant colony algorithm has been widely used in terms of the development of swarm intelligence [9]. Bayesian network was used to model the causal relationship between context information with the probability adopted to represent the uncertain relationships of context information [10]. The authors proposed a DAM4GSM architecture, GSM (Global Sensor Network) as a middleware to collect and process sensor data of intelligent mobile phone [11].

Researches show that effective data storage relies on effective data collection technology, data processing, data mining and intermediate technology, which ensure the validity of data collection and processing, and better utilized by the crowd sensing applications.

\subsection{Incentive Mechanism}

The crowd sensing is achieved in most cases by relying on the user's active participation, but how to effectively attract users to actively participate in large-scale social tasks is a problem for solution in the crowd sensing research.

Ahn and Dabbish first proposed using the game engines to attract groups to complete some difficult work for computer engine [12]. This kind of incentive mechanism is regarded as other crowd-sensing research development foundation $[13,14]$. At present, the crowd sensing also rely on some communities' participation or in bidding and traded on the open market. Participants submit offer to complete a task for the task requirements screening. In addition, the authors also pointed out that groups can cooperate or compete with each other to complete the task by games $[13,15]$. The authors proposed the center platform which requires the user to provide awareness services in mobile crowd sensing system; however, some existing sensing applications and systems lack good incentive mechanism to attract more users to participate in [16]. In order to solve this problem, this paper proposed two kinds of system modes: platformcentric model and user-centric model. As to the platform-centric model, Stackelberg game was used for incentives in this mechanism. Such platform was a leader while users were the followers; as to the user-centric model, it was based on the auctioning incentive mechanism.

Latest researches show that two mainstream types of incentive mechanism of crowd sensing are based on game and auction bidding to attract people to participate therein. Both types of incentives include competition and collaboration. Only effective combination of competition and cooperation will help promote crowd sensing.

\subsection{Crowd Sensing Application}

The crowd sensing concept can be seen from many aspects. With more and more built-in sensor for mobile terminal integrating, the mobile application of intellectual perception is also continuing to expand and has brought tremendous social value.

Using smart Park as an example to summarize the characteristics of crowd-sensing may help it better applied in other fields [17]. Firstly, in the crowd sensing, organizers need to play an important role in the collaborative participation; secondly, the expected participation rate in the design of crowd sensing system is a very important factor to consider; finally, apart from participation in the system's contributors, the crowd sensing system is unable to prevent people with hitchhiking; however, most people are surprised, even if the hitchhikers can also exert beneficial effects on the whole system. The paper explored the context awareness and crowdsourcing trigger relationship in the mobile crowdsourcing systems, analyzed typical context-aware system architecture, and described specific scenarios [18]. The authors proposed the concept of participatory sensing based on Smart-phone [19]. With the development of sensor technology and the growing popularity of mobile devices, users can collect and share information through mobile devices. Combination of these data can be used for social relations, urban computing, real-time traffic monitoring and other exciting new models. The authors described the participatory sensing applications in the environmental monitoring [20]. The paper presented an intelligent transportation system (ITS) architecture based on the crowd sensing 
[21]. With ordinary user's mobile phones as sensors, the user's conscious participation and reporting at local road traffic state may help drivers keep abreast of traffic conditions and road conditions and then make appropriate decisions. Shortcomings of this method lie in that it takes advantage of the user's conscious participation, which spend a lot of time and cost. Researchers both at home and abroad on mobile terminal crowdsourcing application study are constantly continuing. The crowd sensing application will be not just limited to smart traffic, sociality, urban computing and environmental monitoring; therefore, more applications of crowd sensing remain to be mined and explored. We believe that in the near future, the mobile terminal crowd sensing will be in a new way to serve the community.

\subsection{Crowd Sensing Platform}

Researches on the crowd sensing platform research are based on physical layer devices such as sensors, mainly for content providers and developers so as to provide a basic information platform; users can use the computing platform to build their own specific crowd sensing application.

The authors proposed a distributed crowdsourcing platform to ensure the distributed task (with geographic coordinates as the distribution basis) to be assigned to different workers [22]. In this sense, workers send their geographical location to a centralized server, the server will assign task to him according to the location information, and the tasks are distributed by maximum. The paper proposed space crowd sensing platform based on the trust mechanism, which ensures that individuals, groups and communities are constantly involved in the collection, analysis and dissemination of spatio-temporal information, including urban, social and other aspects of information [23]. The paper offered an open platform to record the realtime noise map [24]. At present, the known technologies for recording city noise map have disadvantages of slow update and being expensive; besides, they are based on population and traffic template instead of real time data. The platform is a good solution to noise map in terms of some issues such as lack of integrity and random samples in data collection.

Researches show that most of the platforms are still just for a specific application, but this paper believes the crowd sensing platform's development should have more accessions to applications. Integrate the existing and being developed platforms by various third-party applications so as to provide users with more personalized and comprehensive services.

\section{Conclusion}

In summary, as a new sensing means of Internet of things, the crowd sensing network technology promotes the emergence of a number of innovative applications while also facing a series of new problems and challenges. If we can make more mobile users to participate in the crowd sensing, a wider variety of sensors will generate more massive sensory data, excavate and utilize it effectively; thus the collective wisdom of the masses is bound to play an infinite value.

\section{References}

[1] R. K. Granti, F Ye, H Lei. Mobile crowdsensing: Current state and future challenges[J]. IEEE Communications Magazine, 49(11):32-39(2011).

[2] H. D. Ma, D. Zhao, P.Y. Yuan. Opportunities in Mobile Crowd Sensing[J]. IEEE Communications Magazine, 52(8):29-35(2014).

[3] C. Scaffidi, B. A. Mvers, M Shaw. Intelligently creating and recommending reusable reformatting rules[C]. Proc of the 2009 international conference on intelligent user interfaces. ACM, Florida, USA: [s. n.], pp.297-306(2009).

[4] L. M. Haas, M. A. Hernandez, H Ho. Clio grows up: from research prototype to industrial tool[C]. Proc of the 2005 ACM SIGMOD international conference on management of data. ACM, New York, NY, USA, pp.805-810(2005). 
[5] H. Kang, L. Getoor, B. Shneiderman. Interactive entity resolution in relational data: a visual analytic tool and its evaluation[J]. IEEE TVCG, 14(5):999-1014(2008).

[6] G. G. Robertson, M. P. Czerwinski, J.E. Churchill. Visualization of mappings between schemas $[\mathrm{C}]$. Proc of the SIGCHI conference on human factors in computing systems. ACM, New York, NY, USA, pp.431-439(2005).

[7] T. Dasu, T. Johnson, S. Muthukrishnan. Mining database structure; or, how to build a data quality browser[C]. Proc of SIGMOD conference. ACM, New York, NY, USA, pp.240-251(2002).

[8] V. Raman, J. M. Hellerstein. Potter's wheel: an interactive data cleaning system[C]. Proc of the 27th international conference on VLDB. San Francisco, CA, USA: Morgan Kaufmann Publishers Inc, pp.381-390(2001).

[9] J. P. Yu, X. M. Zhou, M. Chen. Research on representative algorithms of swarm intelligence [J]. Computer Engineering and Applications, 46(25):1-4(2010).

[10]A. Ranganathan, J. AI-Muhtadi, R. H. Campbell. Reasoning about uncertain contexts in pervasive computing environments[J]. IEEE Pervasive Computing, 3(2):62-70(2004).

[11]C. Perera, A. Zaslavsky, P. Christen. Capturing sensor data form mobile phones using global sensor network middleware[C]. Proc of IEEE 23rd international symposium on PIMRC. IEEE, Sydney, Australia, pp.24-29(2012).

[12]L. von Ahn, L. Dabbish. Labeling images with a computer game[C]. Proc of the SIGCHI conference on human factors in computing systems. ACM, New York, NY, USA, pp.319-326(2004).

[13]H. Ma, R. Chandrasekar, C. Quirk. Improving search engines using human computation games[C]. Proc of the 18th ACM conference on information and knowledge management. ACM, New York, NY, USA, pp.275-284(2009).

[14]L. von Ahn, R. Liu, M. Blum. Peekaboom: a game for locating objects in images[C]. Proc of the SIGCHI conference on human factors in computer system. ACM, New York, NY, USA, pp.5564(2006).

[15]L. von Ahn, L .Dabbish. Designing games with a purpose[J]. Communication of the ACM, 51 (8):58-67(2008).

[16]D. J. Yang, G. L. Xue, X. Fang. Crowdsourcing to smartphones: incentive mechanism design for mobile phone sensing[C]. Proceedings of the 18th annual international conference on mobile computing and networking. ACM, New York, NY, USA, pp.173-184(2012)

[17].X Chen, E. Santos-Neto, M. Ripeanu. Crowdsourcing for on-street smart parking[C]. Proc of the second ACM international symposium on design and analysis of intelligent vehicular networks and applications. ACM, New York, NY, USA, pp.1-8(2012).

[18]A. H. Afridi, P. Pakistan. Crowdsourcing in mobile: a three stage context based process[C]. Proc of IEEE ninth international conference on dependable, autonomic and secure computing. IEEE, Sydney, NSW, pp.242-245(2011).

[19] S. S. Kanhere. Participatory sensing: Crowdsourcing data from mobile smart phones in urban spaces[C]. Proc of IEEE international conference on MDM. IEEE, Lulea, pp.3 -6(2011).

[20]V. Kotovirta, T. Toivanen, R. Tergujeff. Participatory sensing in environmental monitoringexperiences[C]. Proc of 2012 sixth international conferences on IMIS. Palermo: [s. n.], pp.155162(2012).

[21]K. Ali, D. Al-Yaseen, A .Ejaz. CrowdITS: Crowdsourcing in intelligent transportation systems [C]. Proc of IEEE Conferences on WCNC. IEEE, Shanghai, pp.3307- 3311(2012). 
[22]L. Kazemi, C. Shahabi. Geocrowd: enabling query answering with spatial crowdsourcing[C]. Proc of 20th international conference on advances in geographic information systems. ACM, New York, NY, USA, pp.189-198(2012).

[23]C. Shahabi. Towards a generic framework for trustworthy spatial crowdsourcing[C]. Proc of the 12 th international ACM workshop on data engineering for wireless and mobile acess. ACM, New York, NY, USA, pp.1-4(2013).

[24]R. K. Rana, C. T. Chou, S. S. Kanhere. Ear-phone: an end-to-end participatory urban noise mapping system $[\mathrm{C}]$. Proc of the 9th ACM/IEEE international conference on information processing in sensor networks. ACM, New York, NY, USA, pp.105-116(2010). 